\title{
Bringing home a piece of our past
}

\author{
The Hayabusa2 spacecraft will soon rendezvous with asteroid Ryugu in order to study its composition using remote \\ sensing, a lander, rovers and sample return, explains Elizabeth Tasker.
}

n 26 February 2018, the Hayabusa2 spacecraft caught sight of its destination for the first time. Asteroid Ryugu appeared as no more than a bright dot on the image snapped by the onboard telescopic camera, but after three years in space it is great to see you are going in the right direction. Meaning 'falcon' in Japanese, Hayabusa2 is an asteroid sample retrieval mission led by the Institute of Space and Astronautical Science (ISAS) at the Japan Aerospace Exploration Agency (JAXA). The mission is the successor to Hayabusa, which visited asteroid Itokawa in 2005.

From June 2018 onward, Hayabusa2 will be close enough to Ryugu to begin to resolve the asteroid's surface features. Then analysis can begin in earnest. One of the first tasks will be to construct a three-dimensional model that can be used to select landing sites and to estimate Ryugu's gravitational field. Even though Ryugu is less than $1 \mathrm{~km}$ in diameter, its weak gravity must be carefully considered for the spacecraft to descend to the surface safely.

The plan for Hayabusa2 is to gather three samples from asteroid Ryugu over a period of the subsequent 12 months and return these to Earth in late 2020: two samples of the asteroid surface, and a collection of subsurface material exposed during a controlled impact. In addition to sample collection, the asteroid will also be explored via a suite of remote sensing instruments, a lander and three small rovers. It is an ambitious itinerary to tackle an equally ambitious science goal: to uncover the original material that initiated life on Earth.

Ryugu is a C-type asteroid, a class of space rock containing carbonaceous material that is thought to have undergone minimal alteration since the Solar System first formed. The small celestial body is therefore kin to the meteorites that pelted the early Earth. This bombardment was a likely delivery service for the Earth's oceans, as our proximity to the Sun would have made it too warm to incorporate solid ices into the forming planet. Moreover, primitive organics have been discovered in meteorites that hint at the delivery of not

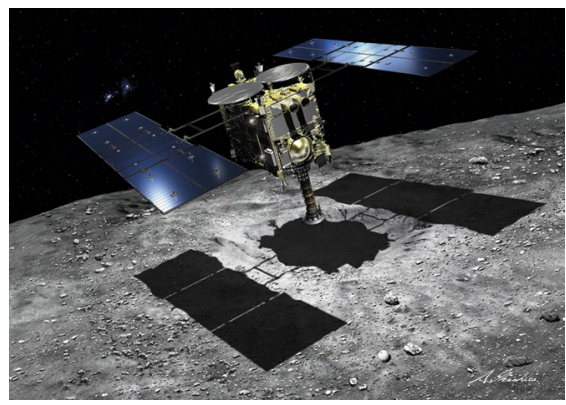

Fig. 1 | An artist's impression of the Hayabusa2 spacecraft collecting a sample from asteroid Ryugu. Credit: (c) JAXA / Akihiro Ikeshita

just the solvent to create life, but also the first ingredients.

Hayabusa2 gathers material through a sampler horn that resembles a long cylinder with a conical end. As the spacecraft touches down on the asteroid, a bullet will be fired into the surface to lift sand, pebbles and fragments of rock into the horn. Should this fail for any reason, the mouth of the horn is equipped with teeth that will raise surface material during touchdown. As the spacecraft decelerates, this regolith will travel up the horn and into the sample container. The sample container has three chambers to store material collected at each landing site on Ryugu. This triple touchdown allows the sampling of different materials that may be present on the asteroid. The third sample collection (Fig. 1) will be particularly distinct because this is when Hayabusa2 will blast a crater on the asteroid surface with its Small Carry-on Impactor (SCI). By gathering from this freshly exposed region, Hayabusa2 will have material that has not been subjected to weathering from the Sun's radiation.

Operations are planned to begin at the asteroid surface in September and October 2018. The current schedule includes the release of the lander, one or two of the rovers and the first sample collection. The second sample collection is currently planned for February 2019 and the third (complete with dropping the SCI) will be in April or May.
A firm schedule has to wait until Hayabusa2 can clearly observe the structure of Ryugu.

The Mobile Asteroid Surface Scout (MASCOT) lander was developed by the German Aerospace Centre and French space agency, CNES - the team behind the Philae comet lander for the ESA Rosetta mission. The lander will analyse the asteroid's regolith using an infrared spectrometer, a magnetometer, a radiometer and a camera, while the rovers will explore an innovative method of transportation in a low-gravity environment: rather than drive, the rovers will 'hop' across the surface. MASCOT will operate for about 15 hours and the rovers for a few days, communicating with the Earth via Hayabusa2.

Hayabusa2 is equipped with a set of three cameras, a near-infrared spectrometer for determining mineral composition, a laser altimeter to measure distance to the surface and a thermal infrared imager. This suite had a test run in December 2015, when the spacecraft flew by the Earth for a gravitational kick on its journey towards Ryugu. The camera filters identified regions of plant life on Earth while the spectrometer detected the presence of water. Once at Ryugu, the same analysis will be performed to search for the presence of hydrated minerals and organic compounds.

Hayabusa2 is not the only mission heading to the asteroids. NASA's OSIRISREx is en route to collect a sample from asteroid Bennu and will begin its approach later this year. The two mission teams are working closely together and will share samples once the spacecraft return. Hayabusa2 is due to return to Earth in 2020, while OSIRIS-REx will arrive three years later. With their arrival, we will have a sample of our origins to explore for years to come.

\section{Elizabeth Tasker}

Department of Solar System Science at ISAS/JAXA, Tokyo, Japan.

e-mail: elizabeth.tasker@jaxa.jp

Published online: 1 June 2018

https://doi.org/10.1038/s41550-018-0486-0 